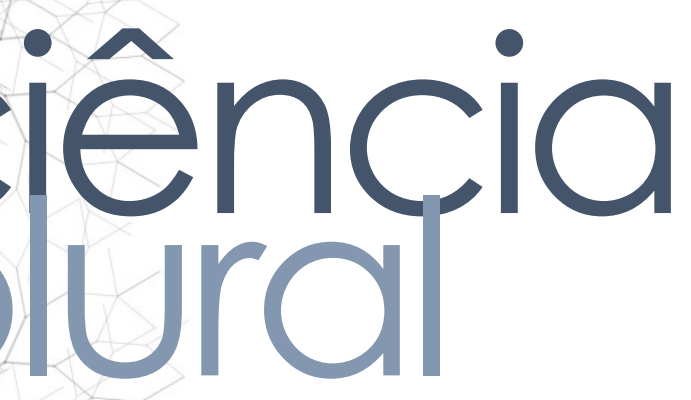

\title{
CAMINHOS PARA O ENVELHECIMENTO SAUDÁVEL: RELAÇÃO ENTRE HIPERTENSÃO ARTERIAL SISTÊMICA E PRINCIPAIS FATORES DE RISCOS MODIFICÁVEIS
}

Pathways to healthy aging: relationship between systemic arterial hypertension and main modifying risk factors

Caminos para un envejecimiento sano: relación entre hipertensión arterial sistémica y principales factores de riesgo modificables

Mayane Carneiro Alves Pereira • Professora Efetiva em Cristo Faculdade do Piauí

- Mestre em Ciência e Saúde pela Universidade Federal do Piauí •

E-mail: mayanealves@hotmail.com

Lúcia de Fátima da Silva Santos • Mestre em Saúde e Comunidade • Universidade Federal do Piauí • E-mail: lucia3584@hotmail.com

Autora responsável pela correspondência:

Mayane Carneiro Alves Pereira • E-mail: mayanealves@hotmail.com 


\section{ciêncíncia
pural}

\section{RESUMO}

Introdução: O Brasil vive um processo de envelhecimento e dentre as doenças mais comuns aos idosos, estão aquelas crônicas não transmissíveis, que apresentam desenvolvimento lento e efeitos deletérios a longo prazo, entre elas, a hipertensão arterial sistêmica, que apresenta como peculiaridade seu caráter multifatorial. Objetivo: Avaliar a relação entre a hipertensão arterial sistêmica e seus principais fatores de riscos modificáveis. Metodologia: Trata-se de estudo transversal de amostragem não probabilística, o levantamento ocorreu junto aos idosos do Programa Terceira Idade em Ação da Universidade Federal do Piauí, no período de agosto a dezembro de 2015. Participaram da coleta, idosos, independente do sexo, devidamente matriculados no programa em questão. Foram excluídos aqueles que apresentavam déficit cognitivo. Cada participante foi avaliado através de um formulário socioeconômico e físico; e avaliação física da circunferência da cintura. Os dados foram analisados por meio do software Statistical Product and Service Solutions versão 20.0. O estudo foi aprovado pelo Comitê de Ética em Pesquisa da Universidade Federal do Piauí. Resultados: Participaram do estudo 99 idosos, sendo 87,9\% do sexo feminino. A maior prevalência de sexo feminino pode estar relacionada ao processo de feminização da velhice, que é caracterizado pela maior expectativa de vida das mulheres. Quanto à atividade física, apenas 15,2\% dos idosos foram considerados praticantes de atividade física regular, sendo que o sedentarismo é um importante fator de risco para doenças crônicas não transmissíveis. Ao avaliar a situação de saúde observou-se que 50,5\% dos pesquisados apresentavam hipertensão arterial sistêmica e correlação significativa com valores elevados de circunferência da cintura $(p=0,03)$. Conclusões: Os hábitos de vida e situação de saúde apontaram que reduzido número de idosos encontram-se ativos; e a relação significativa entre a circunferência da cintura e o diagnóstico de hipertensão arterial sistêmica evidenciam a importância de atuar sobre as diversas variáveis que compõem a saúde.

Palavras-Chave: Hipertensão. Fatores de Risco. Envelhecimento Saudável.

\section{ABSTRACT}

Introduction: Brazil is experiencing an aging process and among the most common diseases among the elderly are chronic non-transmissible diseases, which have slow development and deleterious effects in the long term, including systemic arterial hypertension, which presents its multifactorial character as a peculiarity. Objective: To evaluate the relationship between systemic arterial hypertension and its main modifiable risk factors. Methodology: This is a cross-sectional study of nonprobabilistic sampling, the survey was carried out with the elderly of the Senior Citizens in Action Program at the Federal University of Piauí, from August to December 2015. Elderly, regardless of gender, participated in the collection. enrolled the program in question. Those with cognitive impairment were excluded. Each rticipant was assessed using a socioeconomic and physical form; and physical sessment of waist circumference. The data were analyzed using the Statistical duct and Service Solutions software version 20.0. The study was approved by the earch Ethics Committee of the Federal University of Piauí. Results: This study 
included 99 elderly people, $87.9 \%$ of whom were female. The higher prevalence of females may be related to the feminization process of old age, which is characterized by the higher life expectancy of women. As for physical activity, only $15.2 \%$ of the elderly were considered regular physical activity practitioners, with a sedentary lifestyle being an important risk factor for chronic non-communicable diseases. When assessing the health situation, it was observed that $50.5 \%$ of those surveyed had systemic arterial hypertension and a significant correlation with high waist circumference values $(p=0.03)$. Conclusions: Life habits and health situation showed that a reduced number of elderly people are active; and the significant relationship between waist circumference and the diagnosis of systemic arterial hypertension show the importance of acting on the various variables that make up health.

Keywords: Hypertension. Risk Factors. Healthy Aging.

\section{RESUMEN}

Introducción: Brasil sigue un proceso de envejecimiento y una de las enfermedades más comunes entre los ancianos son las crónicas no transmisibles, de desarrollo lento y nocivo a largo plazo, incluida la hipertensión arterial sistémica, de carácter multifactorial. Objetivo: Evaluar la relación entre la hipertensión arterial sistémica y sus principales factores de riesgo modificables. Metodología: Este estudio transversal de muestreo no probabilístico se realizó por medio de una encuesta con ancianos del Programa "Terceira Idade em Ação" de la Universidad Federal de Piauí, de agosto a diciembre de 2015. No hubo diferenciación de género y aquellos con deterioro cognitivo no participaron del estudio. Cada participante fue evaluado utilizando una encuesta socioeconómica y física, además de evaluación física de la circunferencia de la cintura. Fue utilizado el software de análisis estadístico Statistical Product and Service Solutions versão 20.0 y aprobado por el Comité Ético en Investigación de UFPI. Resultados: 99 personas participaron de la encuesta, el 87,9\% eran mujeres. Esa prevalencia puede estar relacionada con el proceso de feminización de la vejez, caracterizada por la mayor esperanza de vida de este grupo. Cuando el tema es actividad física, solo el $15,2 \%$ de los ancianos eran considerados practicante regular y el estilo de vida sedentario es un conocido factor de riesgo para enfermedades crónicas no transmisibles. Al evaluar la situación de salud, se observó que el $50.5 \%$ de los encuestados tenían hipertensión arterial sistémica y una correlación significativa de altos valores de circunferencia de la cintura $(p=0.03)$. Conclusiones: Los hábitos de vida y la situación de salud mostraron que un número reducido de ancianos están activos y la relación significativa entre la circunferencia de la cintura y el diagnóstico de hipertensión arterial sistémica muestra la importancia de actuar sobre las diversas variables que conforman la salud.

labras clave: Hipertensión. Factores de riesgo. Envejecimiento Sano. 


\section{Introdução}

Nos países em desenvolvimento, a exemplo do Brasil, é considerado idoso todo sujeito com idade igual ou superior a 60 anos. Ao chegar nesta fase, é comum buscar formas de retardar os aspectos negativos do envelhecimento, para isso, é necessário reconhecer quais doenças mais acometem e quais meios podem ser utilizados para frear esse processo. Entre as moléstias mais comuns, está a hipertensão arterial sistêmica (HAS), que é considerada um potencializador do risco de complicações cardiovasculares. Junto a essas mudanças, atualmente, discute-se a proposta de envelhecimento saudável como forma de ampliar a qualidade de vida do idoso, além de auxiliar na prevenção de doenças e na promoção da saúde ${ }^{1}$.

O Brasil, assim como o restante do mundo, vive um processo de envelhecimento que está relacionado a uma melhor condição de vida da população, declínio na taxa de mortalidade e redução nos níveis de fecundidade. A transição epidemiológica, representada pelo envelhecimento, é reflexo de um contexto multidirecional, em que o indivíduo passa por modificações fisiológicas, psicológicas e sociais ${ }^{2}$. Com o decorrer dos anos, as transformações advindas com o envelhecimento passam a ocorrer de maneira mais notável, ou seja, os indivíduos tornam-se menos capazes de enfrentar as alterações do meio ambiente, devido a um maior desgaste orgânico ${ }^{1}$.

Dentre as doenças mais comuns em idosos, estão as Doenças Crônicas Não Transmissíveis (DCNT), que apresentam desenvolvimento lento e efeitos deletérios a longo prazo $^{3}$. Em meio às DCNT, aquelas de origem cardiovascular são as que lideram as causas de óbito no Brasil desde a década de $1960^{4}$. Quanto às manifestações das doenças cardiovasculares, a HAS é a doença mais frequente, atingindo uma prevalência de $65 \%$ entre os idosos do Brasil ${ }^{5}$. Essa enfermidade é definida como uma condição em que os níveis de pressão arterial se apresentam elevados e sustentados ${ }^{6}$. Outra peculiaridade da HAS é seu caráter multifatorial, além do próprio envelhecimento, ela está associada a diversos fatores de risco modificáveis como o cesso de peso, sedentarismo, alimentação inadequada, entre outros ${ }^{6}$.

virtude da mudança nas percepções acerca do envelhecimento, e em meio as mplicações advindas de um envelhecimento não saudável, passou a ser discutido, a ir da década de 1990, o conceito de envelhecimento ativo7 ${ }^{7}$. Por meio de ações que 
estimulem o envelhecimento ativo é possível possibilitar uma vida mais saudável ${ }^{2}$. Considerando os dados expostos, o presente estudo tem o objetivo de avaliar, a relação entre a hipertensão arterial sistêmica e seus principais fatores de riscos modificáveis em idosos.

\section{Metodologia}

Trata-se de estudo com delineamento transversal, que incluiu 99 indivíduos de idade igual ou superior a 60 anos, de ambos os sexos, participantes de um programa de envelhecimento saudável realizado na cidade de Teresina-Piauí, coordenado pela Universidade Federal do Piauí e denominado Programa Terceira Idade em Ação (PTIA/UFPI). No estudo, foi utilizado uma amostragem não probabilística por conveniência, nele, o levantamento dos participante ocorreu por meio de convite nas salas onde ocorriam as aulas do PTIA/UFPI. Após essa identificação, a pesquisadora entrou em contato com cada idoso e agendou uma data para a realização da coleta de dados em uma sala previamente preparada para esse fim, na referida instituição.

No momento da coleta, os indivíduos incluídos no estudo tinham idade igual ou superior a 60 anos, independente do sexo e devidamente matriculados no PTIA/UFPI. Foram excluídos aqueles que apresentavam déficit cognitivo, avaliado por meio do Mini-exame do estado mental. A coleta de dados ocorreu no período de agosto a dezembro de 2015.

Inicialmente, foi realizada uma entrevista com cada participante, conduzida pela pesquisadora, nela, foi lido, com clareza, cada um dos questionamentos propostos no formulário, na tentativa de limitar as incompreensões por parte dos idosos, eliminando possíveis vieses.

No que se refere ao formulário socioeconômico, demográfico e físico, os participantes foram questionados quanto aos dados pessoais (data de nascimento); dados sociais, que incluíram estado civil, sendo agrupados em solteiro(a), asado(a)/união estável, viúvo(a) ou divorciado (a). Quanto ao grau de escolaridade, participantes foram divididos em analfabeto, ensino fundamental (in)completo, ino médio (in)completo ou ensino superior (in)completo. 
Em relação aos fatores de risco, os idosos foram questionados quanto ao diagnóstico de HAS, colesterol LDL (Low Density Lipoproteins) elevado, diabetes mellitus e história familiar de HAS. Não foi especificado, durante o questionário, outras características das doenças, as respostas limitaram-se em sim (apresenta a doença) ou não (não apresenta a doença).

Os participantes também foram indagados quanto ao uso de tabaco e consumo de bebidas alcoólicas. No presente estudo foi classificado como ingestão de bebidas alcoólicas não apenas o seu consumo patológico (etilismo), mas também seu consumo casual, com respostas variando em sim ou não. No item tabagismo os idosos foram divididos em não-fumante, ex-fumante e fumante, segundo o autorrelato. Os participantes foram questionados quanto a presença de outras doenças.

Considerou-se hipertenso aquele indivíduo que autorreferia diagnóstico de HAS e fazia uso de medicamentos anti-hipertensivos a mais de sete dias e/ou indivíduos que, no momento da aferição da pressão arterial, apresentavam PAS superior ou igual a $140 \mathrm{mmHg}$ e PAD superior ou igual a $90 \mathrm{mmHg}$. A aferição da pressão arterial foi realizada em ambos os membros superiores, segundo os critérios propostos pela VI Diretrizes Brasileira de Hipertensão ${ }^{6}$.

No tocante à avaliação da atividade física, os participantes foram classificados em praticantes de atividade física regular e irregular/ausente. Para classificá-los como praticantes de atividade física regular, utilizou-se os dados apresentados pela Sociedade Brasileira de Hipertensão ${ }^{6}$. Segundo ela, para manter uma boa saúde cardiovascular, deve-se realizar, "pelo menos cinco vezes por semana, durante 30 minutos, atividade física moderada de forma contínua ou acumulada, desde que em condições de realizá-la"6. Para o estudo, praticantes de atividade física irregular/ausente foram aqueles que não à realizam, ou realizam em uma frequência inferior à citada.

As medidas de circunferência da cintura foram obtidas com o uso de fita antropométrica, sendo solicitada a elevação da roupa para a exposição da região a ser valiada. A medida foi realizada na região localizada entre as costelas inferiores e a sta ilíaca, no momento da expiração. Foram utilizadas como nota de corte para um cardiovascular aumentado, os valores propostos pela Organização Mundial em 
Saúde $(\mathrm{OMS})^{13}$, que consistiam em valores iguais ou maiores à $80 \mathrm{~cm}$ para mulheres e $94 \mathrm{~cm}$ para homens ${ }^{13}$.

Os dados foram duplamente digitados no Microsoft Excel $\AA$, sendo armazenados com utilização de back-up digital e impressos periódicos. A análise estatística foi realizada utilizando o software Statistical Product and Service Solutions (SPSS) versão 20.0 para Windows. Utilizou-se para análise de normalidade o teste Kolmogorov-Smirnov, considerando significante valores de $\mathrm{p}<0,05$.

Foram calculadas as variáveis resultantes de uma contagem ou mensuração e suas medidas descritas em média e desvio padrão. Utilizou-se o teste Qui-quadrado para verificar associações entre as variáveis qualitativas e teste $\mathrm{t}$ de Student para verificar diferenças médias nas variáveis quantitativas.

O estudo foi submetido e aprovado pelo Comitê de Ética em Pesquisa da Universidade Federal do Piauí (CEP/UFPI), conforme Parecer 1.132.035. Os idosos que aceitaram participar da pesquisa assinaram Termo de Consentimento Livre e Esclarecido aprovado em Comitê de Ética, estabelecido pela Resolução no 466/2012 sobre pesquisa envolvendo seres humanos que atende as exigências éticas e científicas fundamentais. Durante toda a pesquisa, os dados sobre a identificação dos voluntários foram mantidos em sigilo.

\section{Resultados}

\section{Análise Sociodemográfica}

Participaram do estudo 99 idosos, sendo 87 (87,9\%) do sexo feminino e 12 $(12,1 \%)$, do sexo masculino, a idade média dos participantes foi de 68,08 \pm 7,13 anos, variando entre 60 e 89 . A idade média entre os participantes do sexo masculino $(72,25$ $\pm 7,41$ anos) foi superior ao sexo feminino (67,51 $\pm 2,83$ anos).

Quanto ao estado civil, a maioria dos participantes encontram-se casados as)/união estável (51,5\%). A maioria dos entrevistados apresentam níveis mais elevados de escolaridade, atingindo um total de 47 (47,5\%) idosos com ensino superior completo/incompleto. É importante ressaltar que apenas dois participantes rmaram ser analfabetos. 


\section{Fatores de Risco Comportamentais e Situação de Saúde}

Foram analisados os seguintes fatores de risco para as doenças cardiovasculares: tabagismo, ingestão de bebidas alcóolicas e prática de atividade física irregular ou ausente. Quanto à atividade física, 15,2\% dos idosos foram considerados como praticantes de atividade física regular e $84,8 \%$ foram classificados como praticantes de atividade física irregular ou ausente.

Ao investigar a situação de saúde dos integrantes do estudo, constatou-se que 15,2\% autorreferiram diagnóstico de diabetes mellitus e 38,4\% relataram apresentar alteração no nível de colesterol LDL. Ao analisar os valores obtidos com a circunferência da cintura, observou-se que a maioria dos participantes apresentaram valores acima dos níveis de normalidade $(78,8 \%)$, representando um risco cardiovascular elevado, a média obtida foi de $90,1 \mathrm{~cm}( \pm 11,4)$.

As variáveis citadas foram relacionadas ao diagnóstico de HAS (Tabela 1), sendo possível observar uma relação significativa entre a HAS e os valores de circunferência da cintura $(\mathrm{p}=0,03)$.

Tabela 1 - Associação entre diagnóstico de hipertensão arterial sistêmica e situação de saúde dos idosos participantes do estudo e que integram o PTIA/UFPI, Teresina, PI, 2015.

\begin{tabular}{|c|c|c|c|c|c|c|c|}
\hline \multirow[t]{2}{*}{$\begin{array}{l}\text { SITUAÇÃO DE } \\
\text { SAÚDE }\end{array}$} & \multicolumn{2}{|c|}{ HIPERTENSOS } & \multicolumn{2}{|c|}{$\begin{array}{c}\text { NÃO } \\
\text { HIPERTENSOS } \\
\end{array}$} & \multicolumn{2}{|c|}{ TOTAL } & \multirow[t]{2}{*}{$\begin{array}{c}\mathrm{p}- \\
\text { valor }\end{array}$} \\
\hline & $\mathrm{N}$ & $\%$ & $\mathrm{n}$ & $\%$ & $\mathrm{n}$ & $\%$ & \\
\hline DIABETES & & & & & & & 0,377 \\
\hline Sim & 9 & 18,4 & 6 & 12,0 & 15 & 15,2 & \\
\hline Não & 40 & 81,6 & 44 & 88,0 & 84 & 84,8 & \\
\hline COLESTEROL & & & & & & & 0,365 \\
\hline \multicolumn{8}{|l|}{ ELEVADO } \\
\hline Sim & 21 & 42,9 & 17 & 34,0 & 38 & 38,4 & \\
\hline Não & 28 & 57,1 & 33 & 66,0 & 61 & 61,6 & \\
\hline IMC & & & & & & & 0,538 \\
\hline Média (DP) & \multicolumn{2}{|c|}{$26,65(4,4)$} & \multicolumn{2}{|c|}{$25,64(3,73)$} & \multicolumn{2}{|c|}{$26,14(4,09)$} & \\
\hline Baixo Peso & 5 & 10,2 & 9 & 18,0 & 14 & 14,1 & \\
\hline Eutrófico & 26 & 53,1 & 24 & 48,0 & 50 & 50,5 & \\
\hline Sobrepeso & 18 & 36,7 & 17 & 34,0 & 35 & 35,4 & \\
\hline & \multirow{2}{*}{\multicolumn{2}{|c|}{$93(11,47)$}} & \multirow{2}{*}{\multicolumn{2}{|c|}{$87,24(10,77)$}} & & & $0,031^{*}$ \\
\hline Média & & & & & \multicolumn{2}{|c|}{$90,09(11,43)$} & \\
\hline Normal & 6 & 12,2 & 15 & 21,0 & 21 & 21,2 & \\
\hline Risco Elevado & 43 & 87,8 & 35 & 78,0 & 78 & 78,8 & \\
\hline
\end{tabular}

te Qui-quadrado com $5 \%$ de significância. * Relação estatisticamente significativa. C= índice de massa corporal; $\mathrm{DP}=$ desvio padrão; $\mathrm{CC}=$ circunferência da cintura. 


\section{ciência plural}

No estudo, os idosos relataram apresentar 23 tipos diferentes de doenças, entre as mais citadas destacaram-se: HAS $(45,5 \%)$, colesterol elevado $(38,4 \%)$, osteoporose $(19,2 \%)$ e o diabetes mellitus $(15,2 \%)$. Todas as enfermidades foram apresentadas na Figura 1. Quando incluído os pacientes que apresentaram valores de PAS superior a $140 \mathrm{mmHg}$ e PAD superior a $90 \mathrm{mmHg}$, no momento da coleta, a prevalência da HAS elevou-se para $50,5 \%$.

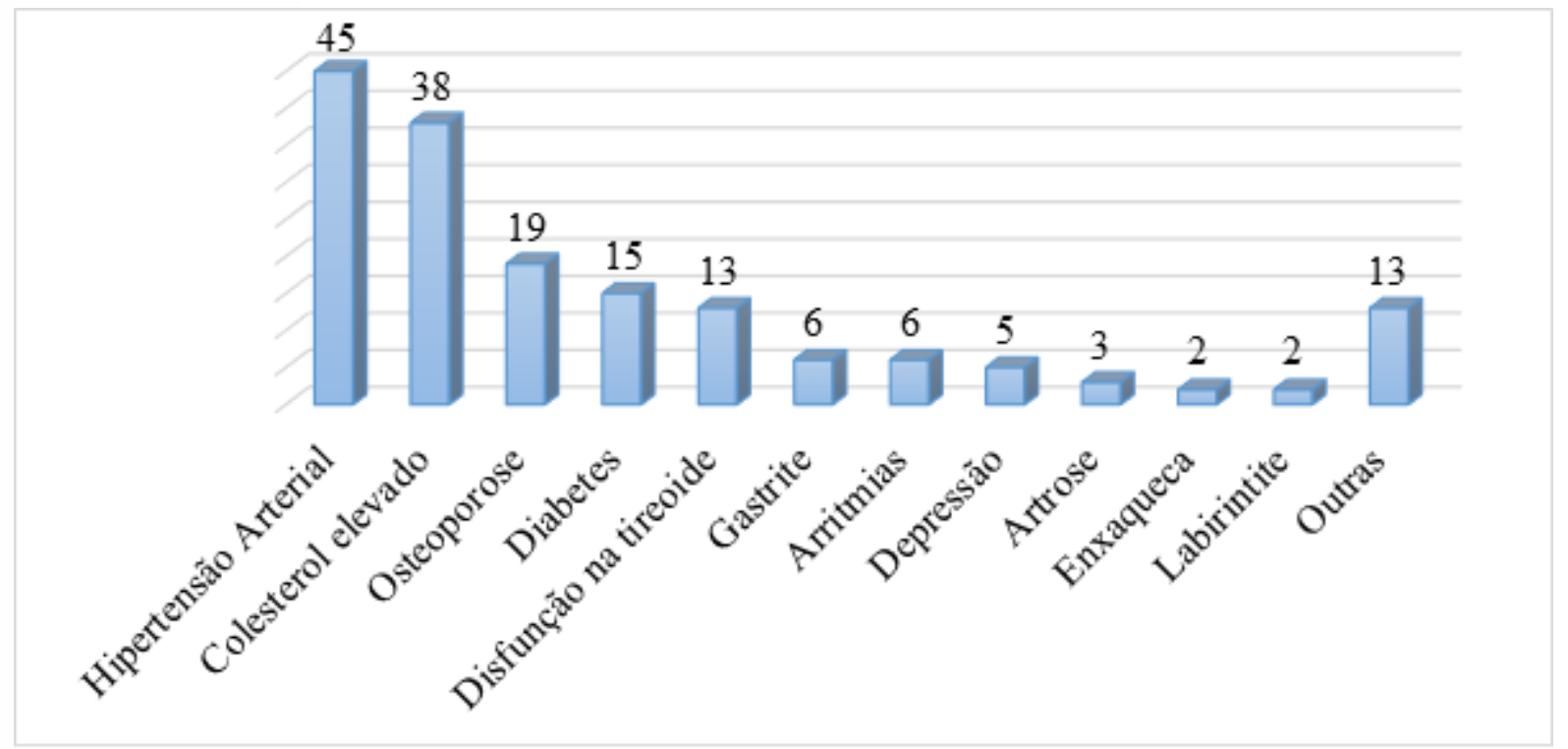

Figura 1 - Distribuição das doenças autorrelatadas pelos idosos participantes do estudo e que integram o PTIA/UFPI, Teresina, PI, 2015.

Nenhum idoso, de idade superior ou igual a 80 anos, relatou estar livre de doenças. Quando relacionada a idade dos entrevistados e o número de doenças, a maioria dos idosos entre 60 e 69 anos e aqueles com idade superior ou igual a 80 anos relataram apresentar, ao menos, uma doença (38,7\% e 63,6\%, respectivamente). A tabela 2 expõe associação entre número de doenças autorrelatadas e faixa etária dos idosos pesquisados. 
Tabela 2 - Associação entre número de doenças autorrelatadas e faixa etária, junto aos idosos participantes do estudo e que integram o PTIA/UFPI, Teresina, PI, 2015.

\begin{tabular}{|c|c|c|c|c|c|c|c|}
\hline \multirow[t]{2}{*}{$\mathrm{N}^{\circ}$ DE DOENÇAS } & \multicolumn{2}{|c|}{60 a 69 anos } & \multicolumn{2}{|c|}{70 a 79 anos } & \multicolumn{2}{|c|}{$\begin{array}{l}80 \text { anos ou } \\
\text { mais }\end{array}$} & \multirow{2}{*}{$\begin{array}{l}\mathrm{p}- \\
\text { valor } \\
0,111\end{array}$} \\
\hline & $\mathrm{n}$ & $\%$ & $\mathrm{n}$ & $\%$ & $\mathrm{n}$ & $\%$ & \\
\hline 0 doenças & 6 & 9,7 & 5 & 19,2 & 0 & 0 & \\
\hline 1 doenças & 24 & 38,7 & 4 & 15,4 & 7 & 63,6 & \\
\hline 2 doenças & 21 & 33,9 & 12 & 46,2 & 2 & 18,2 & \\
\hline 3 ou mais doenças & 11 & 17,7 & 5 & 19,2 & 2 & 18,2 & \\
\hline
\end{tabular}

Teste Qui-quadrado com 5\% de significância.

\section{Discussão}

\section{Caracterização da População}

Inicialmente, é importante destacar a grande diferença em relação ao sexo da população estudada, uma vez que $87,9 \%$ da amostra é do sexo feminino e apenas 12,1\% são do sexo masculino. Situação semelhante foi encontrada em outros estudos realizados junto a idosos que participam de programas de envelhecimento ativo 3,14,15 e em pesquisas compostas por hipertensos ${ }^{16,17}$.

A maior prevalência de idosos do sexo feminino pode estar relacionada a uma diversidade de fatores, como o maior interesse, entre mulheres, de cuidar da própria saúde, o que estimula sua participação em grupos que promovam uma maior qualidade de vida ${ }^{3}$. Outra situação evidente é o processo de feminização da velhice, no qual o Brasil está incluído, e é caracterizado pela maior expectativa de vida das mulheres $3,14,18$.

Na realidade nacional é perceptível o menor interesse da população masculina em cuidar da própria saúde, fato vinculado às características culturais de uma sociedade que não permite ao homem adoecer ou assumir qualquer estado de fragilidade ${ }^{14,15,19}$. Uma das propostas instituídas para modificar esse panorama é a Política Nacional de Saúde do Homem, que tem o intuito de promover, prevenir, ssistir e recuperar a saúde da população masculina, considerando seus determinantes ondicionantes de saúde ${ }^{19}$.

Quanto ao estado civil, foi identificada uma maior prevalência de casados $, 5 \%)$. No entanto, esta variável apresenta-se sem consenso na literatura, variando 
de maior número de participantes $\operatorname{casados}^{3,11,16,20}$ e viúvos ${ }^{14,15}$. Essa oscilação pode estar relacionada às diferentes médias de faixa etária da população de cada pesquisa.

Foi observada uma maior prevalência de idosos com elevado nível de escolaridade (47,5\% apresentam ensino superior completo ou incompleto) e apenas $2 \%$ dos participantes declararam-se analfabetos. Esse resultado contraria os achados da literatura11,12,16. O Instituto Brasileiro de Geografia e Estatística (IBGE) expõe, em sua Síntese de Indicadores Sociais de 2015, uma média de 4,8 anos de estudo entre os idosos brasileiros ${ }^{8}$.

\section{Análise dos Hábitos de Vida}

A adoção de um estilo de vida saudável é essencial para a manutenção da qualidade de vida e o controle dos fatores de risco cardiovasculares ${ }^{6}$. A II Diretrizes em Cardiogeratria trazem recomendações grau I para mudança de estilo de vida entre pacientes hipertensos, as medidas incluem: redução na ingestão de sódio e de álcool, redução do peso corporal, prática de atividade física e suspensão do tabagismo ${ }^{21}$.

No estudo, foram analisados os hábitos de vida dos idosos e sua situação atual de saúde, tais variáveis foram relacionadas ao diagnóstico de HAS. O tabagismo atual foi observado em apenas um participante e 74,7\% dos entrevistados se declararam não fumantes. Semelhante aos dados coletados, Ribeiro et al. (2015) ${ }^{17}$ encontraram uma baixa prevalência de tabagistas (93\% não fumam) em uma população formada por idosos usuários do Programa Hiperdia17; e Basto-Barbosa et al. (2012) ${ }^{11}$ relataram a existência de apenas dois fumantes (3\%) em um estudo com hipertensos de 60 anos ou mais ${ }^{11}$. A pequena parcela de pesquisados fumantes pode ser justificada pelo contexto de mudança de hábitos de vida e cuidados com a própria saúde, comum entre idosos participantes de programas de envelhecimento saudável. Condição confirmada por Silva e colaboradores $(2011)^{15}$, quando buscaram descrever o perfil epidemiológico dos idosos que participam de grupos sociais, nesse caso, o tabagismo foi observado em penas $7 \%$ dos participantes ${ }^{15}$.

O consumo "social" de bebidas alcoólicas está presente em 32,3\% dos trevistados, sem relação significativa com o diagnóstico de HAS. Basto-Barbosa et (2012) observaram que $11 \%$ dos idosos hipertensos são etilistas ${ }^{11}$. No presente 
estudo, foi classificado como ingestão de bebidas alcoólicas não apenas o seu consumo patológico, mas também seu consumo casual. Portanto, a variação nos valores pode estar relacionada ao critério utilizado na avaliação. Essa variação foi verificada na pesquisa de Ribeiro et al. (2015) ${ }^{17}$, nele, apenas 15,2\% dos idosos que frequentam o Programa Hiperdia de Jequié-Bahia são etilista, no entanto, mais da metade já consumiram bebidas alcoólicas em algum momento da vida (52,8\%), uma vez que o ingesta dessas bebidas é uma prática bastante difundida no Brasil ${ }^{17}$.

O sedentarismo é um importante fator de risco para o declínio da capacidade funcional do idoso, com isso, grupos de atividade física surgem como importantes aliados na mudança do estilo de vida e manutenção da independência funcional, retardando complicações advindas do envelhecimento ${ }^{17,20}$. Foi observado que 15,2\% dos idosos encontravam-se praticando alguma atividade física, de maneira regular.

O efeito positivo da atividade física no controle da HAS já é reconhecido, sendo, inclusive, uma das recomendações propostas na VI Diretrizes Brasileiras de

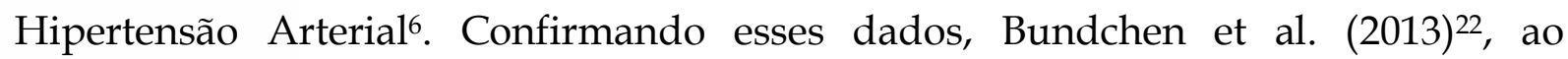
avaliarem idosos hipertensos que realizavam tratamento exclusivo de HAS com atividade física, inferiram que essa prática apresenta efeitos terapêuticos semelhantes ao tratamento medicamentoso ${ }^{22}$. Freire et al. $(2014)^{3}$, em um estudo realizado em Minas Gerais, estimaram que 19,1\% dos idosos praticam exercício físico regularmente, condição semelhante a esta pesquisa. Os autores também ressaltam a importância da proposta de envelhecimento ativo, uma vez que, diminuindo o sedentarismo é possível melhorar e preservar a saúde ${ }^{23}$.

Reconhecendo essa atual necessidade do idosos brasileiros, foi promulgado em 2013, o Decreto $n^{\circ}$ 8.114, que estabelece o Compromisso Nacional para o Envelhecimento Ativo, e tem como finalidade propor a criação de ambientes propícios para a efetivação do envelhecimento saudável, incentivo ao apoio da família e à convivência comunitária e intergeracional; seguindo os eixos da emancipação e protagonismo, promoção e defesa dos direitos, informação e formação ${ }^{24}$. 


\section{Análise da Situação de Saúde}

Quando se trata de doenças cardiovasculares, a HAS não é o único fator preocupante. $\mathrm{O}$ diabetes mellitus, os níveis séricos elevados de colesterol e a obesidade surgem como condições de saúde que ampliam o risco de eventos cardiovasculares, além de aumentar a chance de ocorrência de outras DCNT ${ }^{10}$. Nesta pesquisa, observou-se uma prevalência de $15,2 \%$ de casos de diabetes mellitus e 38,4\% de níveis elevados de colesterol.

Quanto a prevalência de colesterol LDL elevado, os estudos apresentam resultados diversos. Quirós-Meza et al. (2014)25, ao buscar determinar a prevalência de enfermidade ateromatosa e fatores de risco em pacientes ambulatoriais com mais de 50 anos, observaram que a dislipidemia se apresenta como um dos mais presentes fatores de risco primários da aterosclerose ${ }^{25}$. Outros autores encontraram uma prevalência de $28,6 \%$ de colesterol elevado em indivíduos entre 20 e 79 anos que realizam tratamento medicamentoso para controle da $\mathrm{HAS}^{20}$. O excesso de peso representa um risco para a saúde, em especial, quando trata-se de eventos cardiovasculares, tal condição foi verificada nos estudos de Turi et al. (2014)5, ao afirmarem que o excesso de peso, associado ao sedentarismo, representam um dos maiores riscos de HAS5.

Quando avaliada a circunferência da cintura, a média obtida foi de 90,1 \pm $11,4 \mathrm{~cm}$, com um total de $78 \%$ dos participantes com valores acima da normalidade. Por meio das associações realizadas, percebeu-se que a circunferência da cintura apresentou relação significativa com o diagnóstico de HAS $(p=0,03)$, associação relatada na literatura ${ }^{16}$. Confirmando esse achado, a IV Diretrizes de Hipertensão Arterial informa que a perda de peso e de circunferência da cintura estão correlacionadas com menores valores de pressão arterial ${ }^{6}$.

Durante o envelhecimento são vivenciadas alterações corporais, que resultam em uma menor capacidade de adaptação ao ambiente; aumento da vulnerabilidade e incidência de processos patológicos; diminuição da funcionalidade; além do maior arecimento de doenças, em especial as DCNT ${ }^{4}$. Nesse estudo, os idosos relataram resentar 23 tipos diferentes de doenças, dentre elas, a mais comum foi a HAS $5 \%$, seguida do colesterol elevado $(38,4 \%)$, da osteoporose $(19,2 \%)$ e da diabetes 
mellitus (15,2\%). Quando incluído os pacientes que apresentaram valores de PAS superior a $140 \mathrm{mmHg}$ e PAD superior a 90mmHg, a prevalência de HAS elevou-se para $50,5 \%$.

A HAS é uma doença de alta prevalência e baixa taxa de controle no Brasil ${ }^{6}$. Neste País, 65\% dos idosos são hipertensos ${ }^{5}$, esse elevado índice também foi verificado na literatura pesquisada ${ }^{3,16}$. Autores relatam a associação entre a elevada prevalência da HAS à longevidade da população, pois o envelhecimento provoca mudanças no sistema cardiovascular; o que predispõe o aparecimento de doenças como a HAS16.

Semelhante ao presente estudo, algumas pesquisas incluem o colesterol LDL elevado e a diabetes mellitus no grupo das doenças mais frequentes entre os idosos ${ }^{15,18}$, tais moléstias já foram discutidas nesse artigo. A osteoporose foi relatada por 19,2\% dos idosos, uma elevada prevalência de osteoporose e doenças osteoarticulares foram encontradas em outros estudos 9,15,18. A osteoporose é apontada como uma das doenças osteoarticulares mais comum entre idosos, sendo caracterizada pela diminuição da massa óssea, o que ocasiona uma maior fragilidade no idoso, maior risco de fratura, além de facilitar a instalação de incapacidades ${ }^{18}$.

Com o envelhecimento populacional as doenças crônicas vêm substituindo as doenças agudas e, o maior número de doenças crônicas está atrelado a uma maior morbimortalidade entre os idosos ${ }^{9}$. No estudo, a maioria dos idosos relatam apresentar diagnóstico de uma ou duas doenças, que juntas representam 70,8\% da amostra; 18,2\% apresentam três ou mais doenças; e nenhum dos idosos com idade a partir de 80 anos está livre de doenças. Corroborando com esse achado, Virtuoso et al. $(2012)^{18}$, ao avaliar idosos praticantes de atividade física notaram que $87,2 \%$ tem pelo menos uma doença crônica ${ }^{18}$.

\section{Limitações do Estudo}

Destaca-se algumas limitações no presente estudo. Em primeiro lugar, a pesquisa foi realizada exclusivamente junto a idosos que participam de um processo envelhecimento saudável, por meio de uma amostragem por conveniência. Essa uação impede a expansão dos resultados para a realidade de outros grupos que não lizam essa prática. Na pesquisa, utilizou-se o delineamento transversal, método que 
impossibilita o estabelecimento de causalidade entre os desfechos e as variáveis independentes.

Outra limitação observada refere-se ao fato de grande parte das informações serem obtidas por meio do relato dos participantes, situação que facilita a ocorrência de viés de memória. Prova disso é que não foi especificado, durante o questionário, quais as características das doenças, sintomas ou níveis sanguíneos, as respostas limitaram-se em sim (apresenta a doença) ou não (não apresenta a doença). O mesmo ocorreu quando questionados quanto ao uso de tabaco e consumo de bebidas alcoólicas.

\section{Conclusões}

Por meio desse estudo foi possível observar que a maioria dos participantes são do sexo feminino, o que reforça o processo de feminização da velhice. A elevada prevalência de HAS evidenciada nesse estudo reforça o fato de que esta doença representa um risco para a saúde, quando se trata de eventos cardiovasculares fatais e não fatais, além de outras complicações de saúde. Em meio a essa condição, é necessário, por parte do serviço público de saúde, a realização de ações, tanto preventivas quanto paliativas, que permitam o diagnóstico precoce e o tratamento efetivo.

A relação significativa entre os valores elevados de circunferência da cintura e o diagnóstico de HAS ( $p=0,031$ ) evidencia a importância de se avaliar e controlar as diversas variáveis que compõem a saúde. Sendo assim, deve-se estimular, especialmente entre os idosos, a adoção de hábitos de vida saudáveis, como a prática de atividade física regular, o controle de peso corporal, a cessação do tabagismo, a redução na ingesta de bebidas alcoólicas, a adoção de uma alimentação equilibrada, entre outras medidas.

O envelhecimento é um processo amplo e multidimensional, com isso, uma esquisa não é suficiente para pormenorizar todos os seus detalhes. Portanto, são cessários mais estudos que possibilitem ampliar o conhecimento a respeito dos actos da HAS na vida do idoso. 


\section{ciência
piural}

\section{Referências}

1- Miratsuka M, Nakagawa NK. Circulação e Envelhecimento. In: PERRACINI MR, FLÓ CM. Funcionalidade e Envelhecimento. Rio de Janeiro: Guanabara Koogan; 2013. cap. 11.

2- Hernandes NA, Probst VS, Silva Júnior RA, Januário RSB, Pitta F, Teixeira DC. Physical activity in daily life in physically independent elderly participating in community-based exercise program. Braz J Phys Ther. 2013;15(1):57-63.

3- Freitas CMSM, Moura PV, Silva EAPC, Cartaxo HGO, Silva PPC, Caminha IO, et al. Identidade do idoso: representações no discurso do corpo que envelhece. Estudos interdisciplinares sobre o envelhecimento. 2012;17(1):19-35.

4- Ferreira CCC, Peixoto MRG, Barbosa MA, Silveira EA. Prevalência de Fatores de Risco Cardiovascular em Idosos Usuários do Sistema Único de Saúde de Goiânia. Arq Bras Cardiol. 2010;95(5):621-628.

5- Turi BC, Codogno JS, Fernandes RA, Monteiro HL. Prática de atividade física, adiposidade corporal e hipertensão em usuários do Sistema Único de Saúde. Rev. Bras. Epidemiol. 2014 out-dez 17(4):925-937.

6- Sociedade Brasileira de Hipertensão, Sociedade Brasileira de Cardiologia e Sociedade Brasileira de Nefrologia. VI Diretrizes Brasileiras de Hipertensão Arterial. São Paulo. 2010; 13(1).

7- World Health Organization (WHO). Envelhecimento ativo: uma política de saúde. Brasília: Organização Pan-Americana da Saúde. 2005: 60 p.

8- Instituto Brasileiro de Geografia e Estatística (IBGE). Diretoria de Pesquisas. Coordenação de População e Indicadores Sociais. Síntese de indicadores sociais: uma análise das condições de vida da população brasileira. Rio de Janeiro: IBGE. 2015; 137p.

9- Campolina AG, Adami F, Santos JLF, Lebrão ML. A transição de saúde e as mudanças na expectativa de vida saudável da população idosa: possíveis impactos da prevenção de doenças crônicas. Cad. Saúde Pública, Rio de Janeiro. 2013 jun;29(6):1217-1229.

10- Goulart FAA. Doenças crônicas não transmissíveis: estratégias de controle e esafios e para os sistemas de saúde. Brasília, 2011.

Bastos-Barbosa RG, Ferriolli E, Moriguti JC, Nogueira CB, Nobre F, Ueta J, et al. esão ao Tratamento e Controle da Pressão Arterial em Idosos com Hipertensão. . Bras. Cardiol. 2012;99(1):636-641. 
12- Girotto E, Andrade SM, Cabrera MAS, Matsuo T. Adesão ao tratamento farmacológico e não farmacológico e fatores associados na atenção primária da hipertensão arterial. Ciência \& Saúde Coletiva. 2013;18(6):1763-1772.

13- World Health Organization (WHO). Obesity: Preventing and managing the global epidemic. Geneva; 1997.

14- Andrade AN, Nascimento MMP, Oliveira MMD, Queiroga RM, Fonseca FLA, Lacerda SNB, et al. Percepção de idosos sobre grupo de convivência: estudo na cidade de Cajazeiras-PB. Rev. Bras. Geriatr. Gerontol. 2014;17(1):39-48.

15- Silva HO, Carvalho MJAD, Lima FEL, Rodrigues LV. Perfil epidemiológico de idosos frequentadores de grupos de convivência no município de Iguatu, Ceará. Rev. Bras. Geriatr. Gerontol. 2011;14(1):123-133.

16- Esperandio EM, Espinosa MM, Martins MSA, Guimarães LV, Lopes MAL, Scala LCN. Prevalência e fatores associados à hipertensão arterial em idosos de municípios da Amazônia Legal, MT. Rev. Bras. Geriatr. Gerontol. 2013;16(3):481-493.

17- Ribeiro RM, Tribess S, Santos AS, Pinto LLT, Ribeiro MCL, Roza LB, et al. Barreiras no engajamento de idosos em serviços públicos de promoção de atividade física. Ciência \& Saúde Coletiva. 2015; 20(3):739-749.

18- Virtuoso JF, Mazo GZ, Menezes EC, Cardoso AS, Dias RG, Balbé GP. Perfil de morbidade referida e padrão de acesso a serviços de saúde por idosos praticantes de atividade física. Ciência \& Saúde Coletiva. 2012;17(1):23-31.

19- Brasil. Ministério da Saúde. Secretaria de Atenção à Saúde. Departamento de Ações Programáticas e Estratégicas. Política Nacional de Atenção Integral à Saúde do Homem: princípios e diretrizes. Brasília, DF, 2009, 92 p.

20- Costa M, Rocha L, Oliveira S. Educação em saúde: estratégia de promoção da qualidade de vida na terceira idade. Revista Lusófona de Educação. 2012;22:123-140.

21- Sociedade Brasileira de Cardiologia. II Diretrizes Brasileiras em Cardiogeriatria. Arq Bras Cardiol. 2010;95,1-112.

22- Bundchen DC, Schenkel IC, Santos RZ, Carvalho T. Exercício físico controla pressão arterial e melhora qualidade de vida. Rev Bras Med Esporte. 2013;19(2):91-95. gular de atividade física: estudo de base populacional no norte de Minas Gerais, rasil. Rev Bras Med Esporte. 2014;20(5):345-349.

Brasil. Decreto 8.114, de 30 de setembro de 2013. Estabelece o Compromisso ional para o envelhecimento ativo e institui comissão interministerial para 
monitorar e avaliar as ações em seu âmbito e promover a articulação de órgãos e entidades públicas envolvidas em sua implementação. Diário Oficial da União, 1 out 2013.

25- Quirós-Meza G, Salazar-Nassar J, Castillo-Rivas J, Vásquez-Carrillo P, MirandaÁvila P, Fernandez-Morales H. Prevalencia y factores de riesgo de enfermedad ateroesclerótica sistémica. Acta méd costarric. 2014;56(1):6-11. 